\section{Multidisciplinary SCIENTIFIC JOURNAL OF MARITIME RESEARCH}

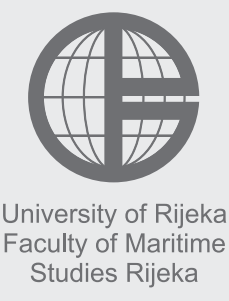

\section{Multidisciplinarni \\ znanstveni časopis \\ POMORSTVO}

\title{
Ship systems for natural gas liquefaction
}

\author{
Aleksandar Vorkapić, Predrag Kralj, Dean Bernečić \\ University of Rijeka, Faculty of Maritime Studies Rijeka, Studentska ulica 2, 51000 Rijeka, Croatia, e-mail: pkralj@pfri.hr
}

\section{ABSTRACT}

This review describes the current liquefaction systems on ships for natural gas transportation in liquid form (Liquefied Natural Gas - LNG). Since in the construction of a gas carrier an essential requirement is the selection of a liquefaction system, this paper describes the principles of operation, thermal and technological processes, as well as the advantages of each system.

\section{ARTICLE INFO}

Review article

Received 2 November 2016

Accepted 12 December 2016

Key words:

Liquefaction

Liquefied natural gas

Brayton process

Cascade process

\section{Introduction}

Evaporation of natural gas (boil-off gas $\mathrm{BOG}^{1}$ ) is inevitable due to difference in temperature between cargo and the ambiance i.e., air and sea. The movement of the cargo inside the tank further assists evaporation during the voyage. Cargo movement depends on the ship's size, tank design and weather and sea conditions.

Ships for the transport of liquefied natural gas, built up to 2005 , were mostly equipped with a steam turbine as the main propulsion engine. Boil-off gas is collected in the tank and burnt in the steam generators. Thermal energy of the steam drives the steam turbines, which, through a gear, drive the ship's propeller and steam turbine generators to produce electricity. Between 2006 and 2010, when gas prices reached their historically high values, combustion of boil-off gas cargo in low efficient steam-turbine plants has become economically unprofitable. Parallel development of slow-rotating two-stroke diesel engines, which are becoming economically proven, and the development of more compact and more cost-effective liquefaction, is leading to

1 Cargo vapors created by heat transfer from the environment onto the cargo transported in liquefied form (while maintaining a constant pressure in the reservoir) the construction of ships for the transport of liquefied natural gas with modern diesel-engine propulsion systems that burn heavy fuel and built-in systems for liquefaction of boiloff cargo. Changes do not stop there, because fluctuations in the prices of heavy fuel and gas in the last decade and more stringent legislation concerning the discharges of pollutants from combustion into the atmosphere are making way for diesel engines able to burn gas or heavy fuel.

Company Qatargas has, during its expansion, in partnership with various shipping companies, built 45 LNG carriers, intended for longer journeys and driven by diesel-engines fueled by the latest generation of heavy fuel (MAN-ME) with integrated liquefaction of cargo vapor. Two series of ships were built, one was called Q-max, with capacity of approximately $266,000 \mathrm{~m}^{3}$ and equipped with the liquefaction system Cryostar EcoRel, and the other slightly smaller Q-flex, with capacity of approximately $216,000 \mathrm{~m}^{3}$ and equipped with Hamworthy MARK-I and MARK-III type liquefaction systems.

TGE by Tractebel can also be found in use and different combined systems of gas combustion in the dual fuel diesel engines.

Natural gas is a mixture of gases, among which the most abundant is methane (Table 1). Other gases include 
ethane, propane, butane and nitrogen which is present in an amount of up to $1 \%$, depending on the gas origin. Boiling point of nitrogen at atmospheric pressure is $-196^{\circ} \mathrm{C}, 35^{\circ} \mathrm{C}$ lower than the boiling point of methane at $-161{ }^{\circ} \mathrm{C}$. High content of non-condensable gases in the boil-off gas increases the pressure in the process and thus reduces the liquefaction process efficiency [1].

Table 1 Composition of natural gas [2] and the composition of the boiloff gas - BOG [3]

\begin{tabular}{|l|c|c|}
\hline \multicolumn{1}{|c|}{ Component } & Mol \% NG & Mol \% BOG \\
\hline Methane & $87.0 \div 98.0$ & Min $91.0 \%$ \\
\hline Ethane & $1.4 \div 9.5$ & Max $0.01 \%$ \\
\hline Nitrogen & $0.1 \div 1.0$ & Max $9.0 \%$ \\
\hline Propane & $0.4 \div 2.5$ & In traces \\
\hline Butane & $0.1 \div 0.5$ & $0.0 \%$ \\
\hline Pentanes and heavier gases & $0.0 \div 0.1$ & $0.0 \%$ \\
\hline
\end{tabular}

By boiling-off of light components (nitrogen and methane) the cargo in the tank changes its composition. This phenomenon is called aging, and it is important in the evaluation of natural gas because its price on the market depends on the energy value of the delivered gas. If the cargo vapors are burnt in steam generators, then the quality of the cargo decreases in the proportion to the transportation time.

Boil-off of cargo is usually represented with an evaporate percentage of the total volume of cargo in 24 hours or the boil-off rate (BOR). For boil-off conditioned by heat transfer BOR is 0.10 to $0.15 \%$ for ships with cargo and 0.06 to $0.10 \%$ for ships under ballast [4].

\section{Thermodynamic basics and optimization of natural gas liquefaction}

Liquefaction system which is installed on ships for transport of natural gas usually consists of a:
- closed refrigeration cycle and

- cargo cycle.

In a closed refrigeration cycle, mixed refrigerants (MR) in the Joule-Thomson circle (JT) are generally effective in reducing the difference in temperature with a small number of built-in components. On the other hand such mixtures are hard to place onboard ships, and this is why pure cooling media are exclusively represented in the ship's systems for the liquefaction of natural gas.

Pure media are easy to use, but they need a larger number of degrees of cooling. Research has shown that the reverse Brayton nitrogen cycle is the optimal choice of a closed refrigeration cycle for ship's systems. The advantages are the robustness of operation, low acquisition price and the possibility of producing nitrogen in ship's generators of nitrogen, non-flammability and safe operation at pressures over 100 bar [5]. The disadvantages are that an expansion turbine cannot operate in a two-phase area (liquefied and gaseous phase). In case of partial liquefaction when drops enter the turbine blades turning at peripheral speed of $200-300 \mathrm{~m} / \mathrm{s}$, turbine may suffer permanent damage. Therefore, when designing a process care must be taken to ensure that the liquid phase never enters the turbine. The consequence is that the Brayton cycle cannot produce liquid, and cooling effect of the cycle is thus limited and boil-off temperature control reduced. Another problem is that the turbine, even with the smallest size of the rotor diameter, requires a significant coolant flow, which reduces the lower limit of the cooling capacity of the system to about $500 \mathrm{~W}$. This limitation, however does not affect the shipboard installations.

Since natural gas is dominantly a mixture of hydrocarbon gases, the specific heat is variable with temperature at various points in the liquefaction process and it is therefore important to reduce the entropy generated by the temperature difference $(\Delta \mathrm{T})$ in a cryogenic heat exchanger [6].

Nitrogen refrigeration cycle, as shown in the T-s diagram in Figure 1 comprises four reversible processes: adi-
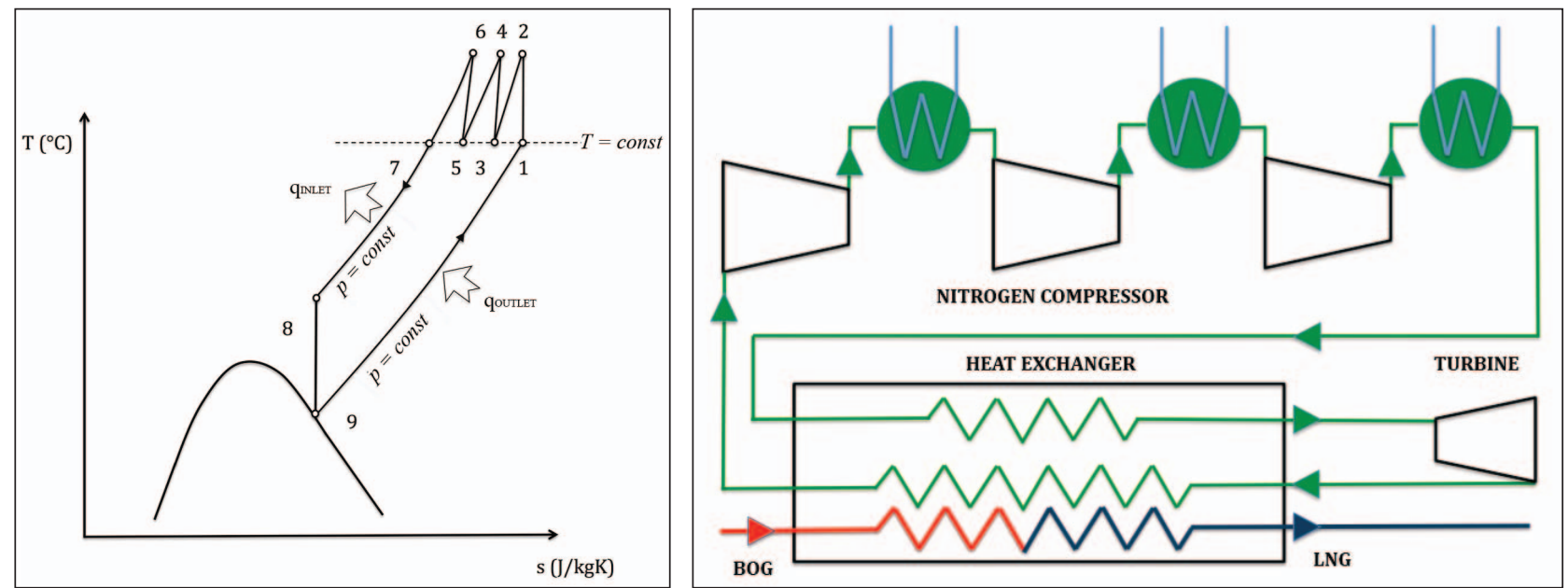

Figure 1 Brayton closed nitrogen refrigeration cycle 

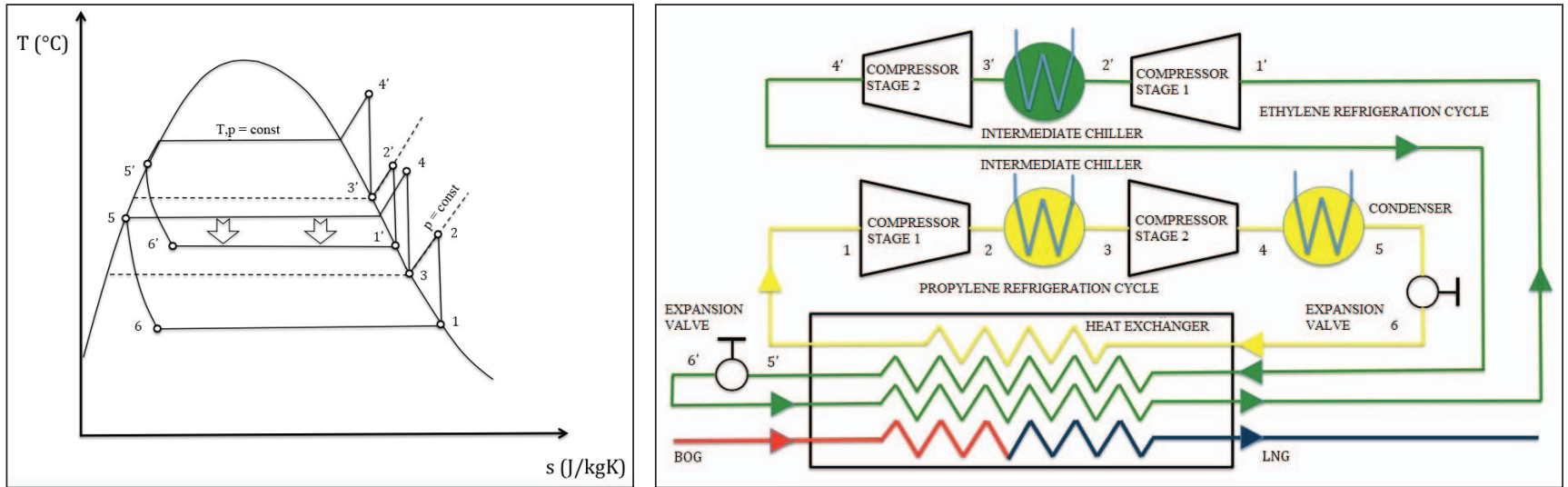

Figure 2 Cascade refrigeration cycle of propylene and ethylene

abatic compression of nitrogen in three stages with heat removal in coolers after each stage (1-7), additional cooling of gas at constant pressure (7-8), adiabatic expansion in the cryogenic expansion turbine (8-9) and expansion of cargo at constant pressure (9-1). After the first and second stage of compression gas passes through intermediate coolers $(2-3 ; 4-5)$, and after the third compression stage through a cooler (6-7). Compander consists of a threestage compressor driven by an electric motor on the same axis with the cryogenic turbine, in which the expansion of gas performs a partial recuperation of consumed energy and thereby reduces the consumption of electricity for running the compressor.

Alternative to the Brayton nitrogen refrigeration cycle is a combination of ethylene and propylene in a cascade refrigeration cycle [1].

Liquefaction process consists of three cycles: natural gas, ethylene (C2) and propylene (C3). Closed ethylene and propylene cycles have two compression stages, while the natural gas is compressed in three stages to reduce the temperature differential in the exchanger. Figure 2 shows the closed refrigeration cycles C2 and C3 which consist of the adiabatic compression in two stages (1-2 and 3-4), with intermediate cooling at constant pressure (2-3), condensation (4-5 at C3), expansion in the expansion valve - Joule Thompson expansion (5-6) along the constant enthalpy curve and heat transfer at constant pressure in the main heat exchanger (6-1).

Thermodynamic efficiency of the primary Brayton cycle can be improved by installing an additional expansion turbine and a pre-cooler [6]. Joule-Thomson expansion is suitable for the cycle in which it is necessary to achieve lower temperatures, while the Brayton principle with the expansion turbine is thermodynamically more efficient. Claude's cycle is a combination of turbine expansion and damping and achieves excellent results in liquefaction of natural gas. Removal of the third heat exchanger after the nitrogen compressor produces the Kapitza cooling cycle which is characterized by reduced cost and improved operability [7].

\section{Liquefaction systems}

In designing the ship's liquefaction systems, when choosing a suitable system the following conditions should be taken into account:

- previous experience with the liquefaction technology

- possibilities of sudden starting up and stopping

- ease of control

- possibility of using in specific conditions of navigation (rolling, pitching)

- limited space for installation

- price

- ease of installation

- safety of crew, environment, cargo and ship

- prescribed redundancy

In addition to the above main requirements, there are still four additional operating parameters that affect the selection:

- liquefaction pressure of boil-off cargo

- temperature of boil-off gas

- cargo system

- system capacity

\subsection{Cryostar ecorel}

After 15 month of intensive testing by the Exxon-Mobil Qatargas Ltd. it was decided that the Ecorel system by Cryostar is suitable for installation on up to that time the largest LNG ships of the Q-max class.

Despite the fact that the technology is generally known, the challenges were limited dimensions and system control in order to accommodate for use in onboard requirements. Ship's conditions are specific according to changes of load, stopping and starting the system, weather conditions and spraying from the top of the tank. Liquefaction with terrestrial plants is mostly under continuous load and typically does not stop except for maintenance [8].

The EcoRel liquefaction system, shown in Figure 3, is based on the principles of the Brayton cycle with a closed refrigeration cycle and capacity proportional to power necessary to re-liquefy the boil-off cargo, and is regulated 


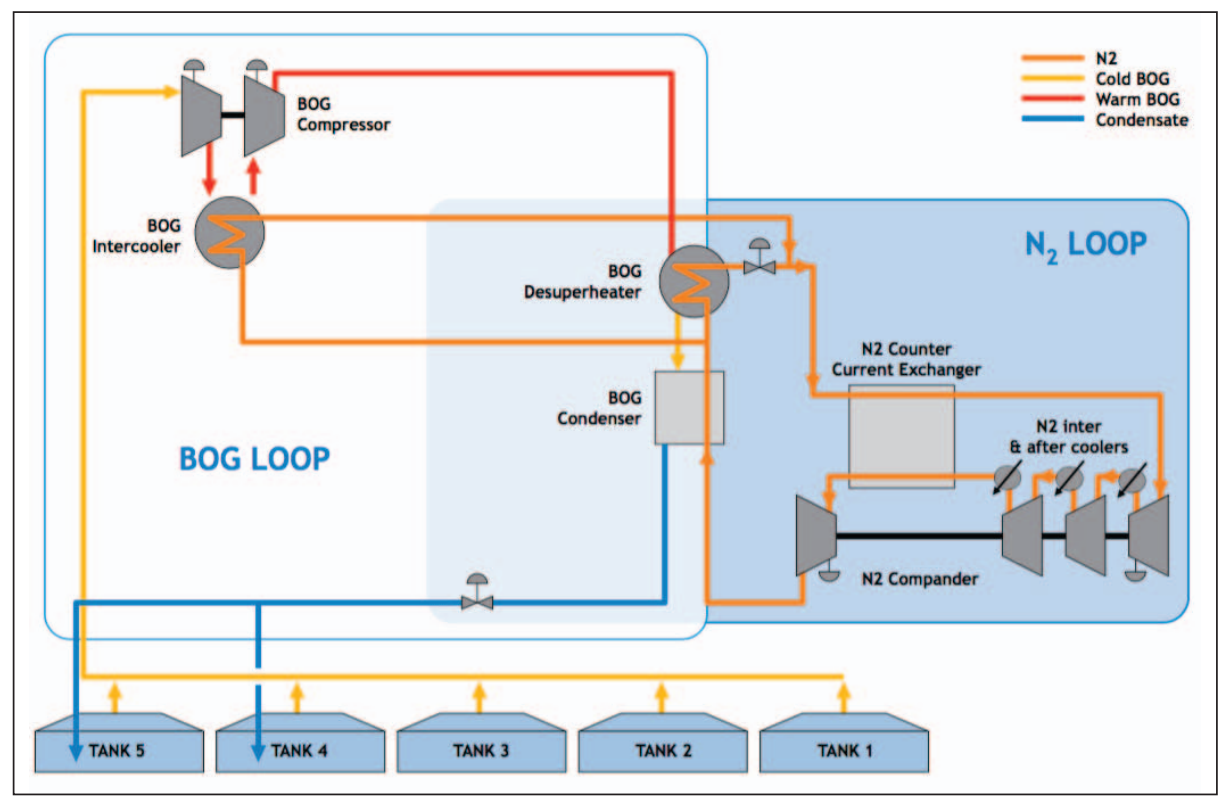

Figure 3 EcoRel system with two-stage compression of boil-off cargo [8]

by the flow of the refrigerant (nitrogen). Capacity is $7 \mathrm{t} / \mathrm{h}$ with compander power of of about 5.2MW [8]. BOG is pumped into a two stage centrifugal compressor at a pressure of 4.8 bar. BOG is cooled in the intermediate cooler between two stages of compression by a stream of nitrogen after the main heat exchanger. Before entering the main exchanger, BOG passes through a de-superheater that protects the main heat exchanger from excessive temperature difference. Condensing temperature depends on the composition of the boil-off cargo and the pressure at which condensation is performed.

In addition to a refrigeration system with three-stage nitrogen compressor and pressure of $47 \mathrm{bar}$, and intermediate cooler and a cooler at the outlet of the third stage the temperature of the nitrogen of $-105^{\circ} \mathrm{C}$ and a cryogenic expansion turbine (nitrogen expands at 9.5 bar while the temperature is lowered to $-168^{\circ} \mathrm{C}$ ) which together make a compander, the refrigerant system also has a countercurrent heat exchanger [9]. Its task is to increase the cooling system efficacy.

\subsection{Hamworthy MARK- I and MARK- III}

System MARK-I, as depicted in Figure 4, consists of two main cycles: open cycle of cargo liquefaction and closedcircuit nitrogen refrigeration cycle [10]. Capacity is $6 \mathrm{t} / \mathrm{h}$ with energy consumption at full capacity of about 5.8 MW (9).

Nitrogen refrigeration cycle consists of the following elements: two N2 companders, water cooled cooler, cold boxes (thermally insulated Hamworthy heat exchanger and separator is called the cold box (CB)), nitrogen tank, two nitrogen compressors, dryer and control and shutoff valves.

Cargo liquefaction cycle consists of: one BOG preheater, two BOG compressors (100 \% redundancy), main heat ex- changer (part of the cold box), liquid and gas phase separator (part of the cold box) and two LNG transfer pumps.

The mixture of the vaporized gas from the cargo tanks at a temperature of about $-100^{\circ} \mathrm{C}$ and the overpressure between 1.06 and 1.12 bar is fed into a two-stage centrifugal compressor. Not pictured, but often a pre-cooler is installed before the compressor cooled by liquefied gas at the outlet of the separator which is used to pre-cool the BOG to a temperature of $-120^{\circ} \mathrm{C}$. Heat exchanger is designed in a way that enables the separation of any heavier liquefied component in order to protect the compressor from the hydraulic hammering. The load is controlled by increasing or decreasing mass quantity of nitrogen in the cycle [11].

In the heat exchanger the nitrogen refrigeration cycle takes heat from the boil-off cargo and turns it into a liquid. In is separator, depending on the pressure, gases that have not been liquefied are being separated. Depending on the plant, non-flammable gases can burn in the burner (Gas Combustion Unit- GCU) or go back into the tanks. It is mostly nitrogen liquefaction temperature which is lower than that of hydrocarbon gases.

At the downstream part is a cargo pump whose task is to overcome the pressure in the tank if it is higher than the one in the heat exchanger.

Cryogenic temperature was kept constant by increasing and decreasing the cooling capacity of nitrogen from 0 to $100 \%$ of settings. Nitrogen in the compressor compresses from 13.2 bar to 53.1 bar with the removal of heat in the coolers between the first and second stage and between the second and third stage, and the cooler after the third stage of compression. The temperature at the exit of the cooler is constant, at $41^{\circ} \mathrm{C}$. High-pressure nitrogen at the temperature of around $40{ }^{\circ} \mathrm{C}$ is fed into the warmer end of the exchanger where it cools down to $-110^{\circ} \mathrm{C}$, giving off heat to the low-pressure nitrogen. 


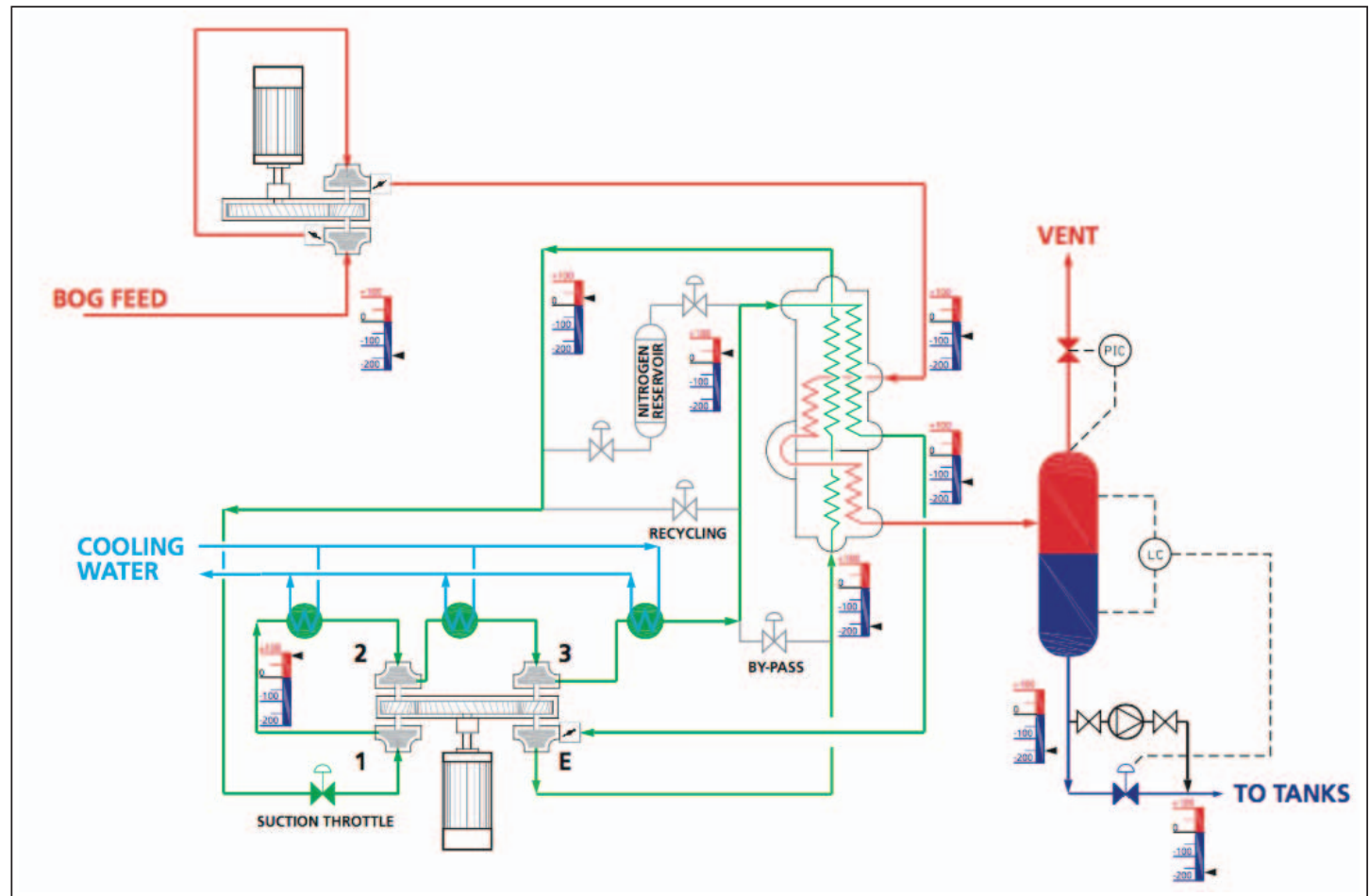

Figure 4 Hamworthy MARK I cargo liquefaction system [10]

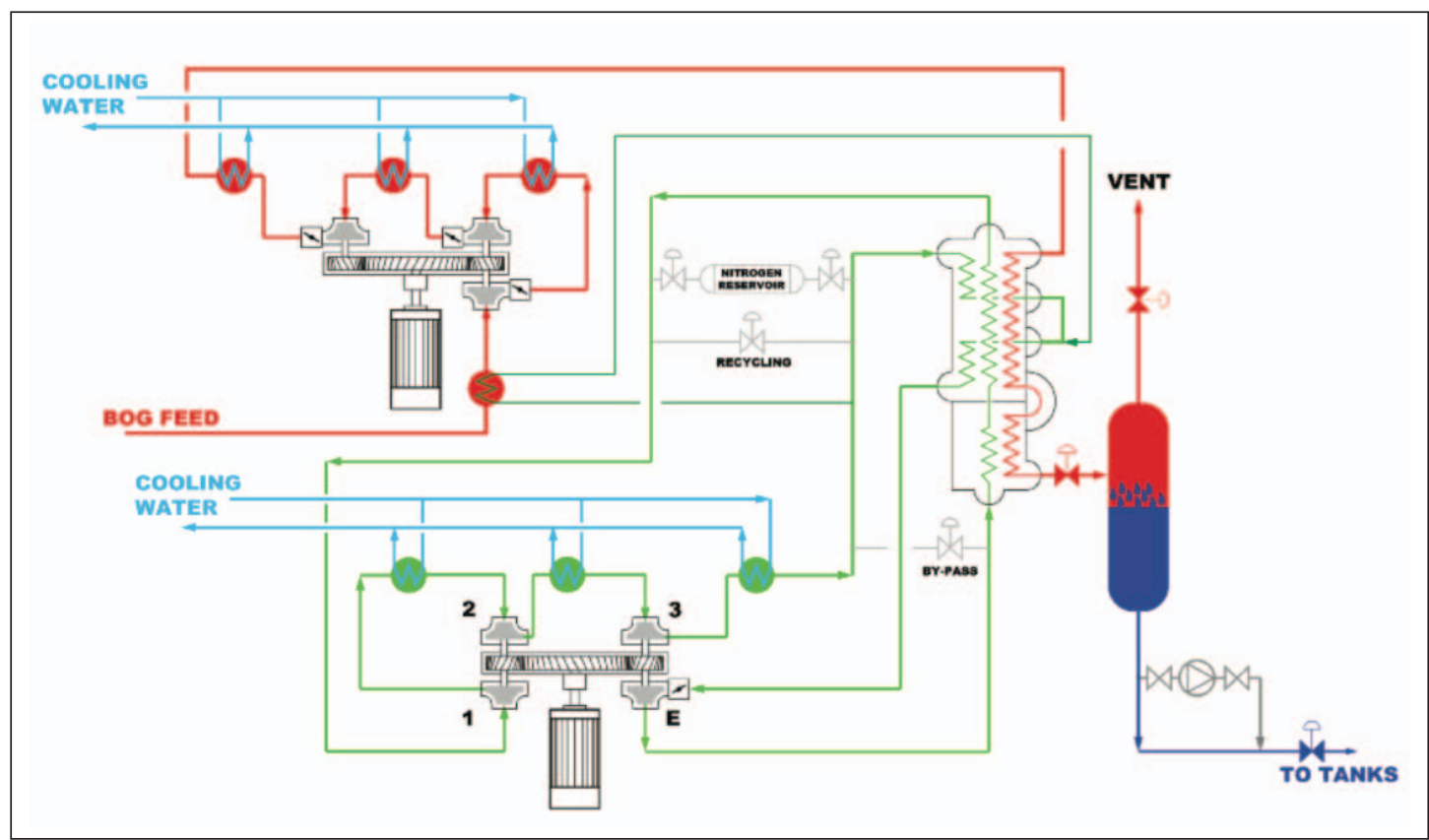

Figure 5 Hamworthy MARK III cargo liquefaction system [10]

Then it goes to the expander where the pressure is 13.2 bar and the temperature $-162.5^{\circ} \mathrm{C}$, from where it goes back to the main heat exchanger and extracts heat from the cargo and the high pressure nitrogen thus closing the cycle [11].

The control system switches modes based on the pressure in the cargo tanks. Sensors and transmitters supply the necessary signals to be processed in the programmable controller.
The first generation plant has been replaced by an improved series MARK III (Figure 5) which, along with additional compression ratio of boil-off cargo, intermediate coolers and the preheater instead of a pre-cooler, consumes up to $15 \%$ less energy for the same cooling effect [10]. Incorporating three-stage compressor that works at ambient temperature, instead of the two-stage cryogenic one in the MARK-I version, reduces costs and overall dimensions of the installation. 
Compressed nitrogen from the heat exchanger passes the preheater at the entrance to the three-stage compressor of boil-off cargo where its temperature is raised from $-100{ }^{\circ} \mathrm{C}$ to $37^{\circ} \mathrm{C}$. Better cooling effect is achieved by the installation of an additional compression stage, which allows heat removal and condensation of the boil-off cargo at higher temperatures and at a pressure of 8 bar (almost twice higher than that of the MARK-I system), as well as with the earlier removal of the of compression heat in the intermediate coolers between the compression stages, and in the cooler after the third stage. The temperature after the intermediate cooler and at the outlet of the cooler after the third stage was maintained constant at $41{ }^{\circ} \mathrm{C}$ [11].

In the nitrogen refrigeration system at $100 \%$ of capacity the three-stage compressor compresses approximately $90,000 \mathrm{~kg} / \mathrm{h}$ of nitrogen from about 10 bar to 42 bar with removal of heat by cooling water to maintain the temperature at $41{ }^{\circ} \mathrm{C}$ [11]. Compressed nitrogen is divided into two streams, one leading to the top of the cold box and the other removing the heat from the boil-off cargo in the preheater. After that the two streams meet and enter the main exchanger where they are cooled to $-110^{\circ} \mathrm{C}$ [9].

The cold stream of nitrogen (after expansion) enters the main heat exchanger at a pressure of about 10 bar and takes away the heat from the boil-off cargo and the two nitrogen streams at a higher pressure. $1000 \mathrm{~kW}$ is generated during the expansion and transmitted back to the compression system which reduces consumption of the electric motor. Low-pressure nitrogen leaves the exchanger at a temperature of $39.5^{\circ} \mathrm{C}$ and pressure of 9 bar [3]. Capacity of the Mark III system is $7 \mathrm{t} / \mathrm{h}$ with power consumption at full capacity of about 5.5MW [9]. This can be installed on the Q max and Q flex size of the ship [9].

\subsection{Tractebel TGE}

TGE liquefaction system, like the one on Figure 6, consists of two cycles: refrigerant nitrogen and cargo and is design for installation on a ship to transport liquefied natural gas capacity of $228,000 \mathrm{~m}^{3}$ [3].

The system has a capacity of $6,250 \mathrm{~kg} / \mathrm{h}$ with a cargo density of $470 \mathrm{~kg} / \mathrm{m}^{3}$, and the boil-off rate of 0.14 . In reality, the boil-off rate is lower, in the range between 5,500 and $5,800 \mathrm{~kg} / \mathrm{m}^{3}$, depending on its composition. The main cryogenic heat exchanger has $25 \%$ while the plant has about $5 \%$ redundancy [3].

When designing the system redundancy is taken conservatively because the plants are very carefully calculated and any increases in power can lead to major changes in the size, power consumption and cost of installation.

Within the refrigerant nitrogen cycle the pressurization is carried out in three stages of compression up to the final 3-6 bar. The third stage is mechanically connected with a turbo expander, which returns $1200 \mathrm{~kW}$ of produced energy back into the process and thus increasing the energy efficiency. Approximately $700 \mathrm{~m}^{3}$ of refrigerant sea of temperature of $32{ }^{\circ} \mathrm{C}$ with a $6{ }^{\circ} \mathrm{C}$ rise in temperature after each cooler is necessary to cool the compressed nitrogen in the coolers and the cooler after the expander [3].

The main heat exchanger is of a three-way type and is used for condensing the boil-off cargo by transferring the heat to the nitrogen after the expander. In order to increase the cooling effect of the cycle, the compressed nitrogen stream passes through a heat exchanger transferring the heat to the expanded nitrogen.

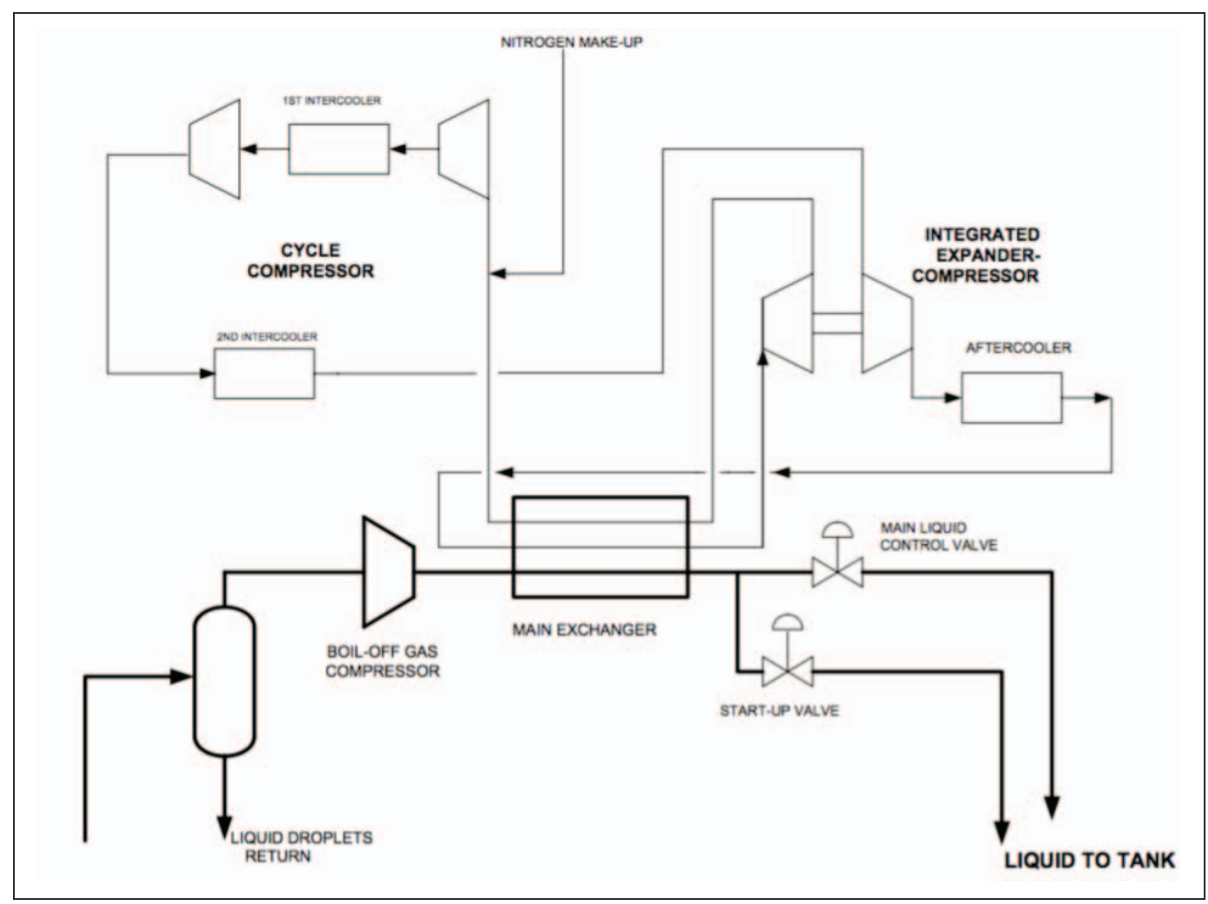

Figure 6 TGE Boil-off Re-liquefaction Unit [3] 


\section{Combined liquefaction systems with combustion within the drive unit}

With the introduction of more stringent environmental regulations concerning the emission of harmful substances into the atmosphere and the increase in the prices of heavy fuel, the use of liquefied natural gas as fuel for combustion in dual fuel diesel plants is imposed as an acceptable alternative with an expected increase of usage in the future. Dual fuel diesel engine appears in two versions: propulsion slow-rotating two-stroke dual fuel diesel engine and medium rotating four-stroke dual fuel diesel engine with or without electric propulsion.

The following two solutions are generated due to a compact compressor Laby-GI developed by the company Burchhardt Compression as well as the development of the bi-stroke engines by MAN Diesel \& Turbo with combustion of natural gas and diesel fuel.

MAN Diesel \& Turbo (MDT) cooperates with several manufacturers of liquefaction system, so it is possible to find MAN ME-GI with the MARK-III liquefaction system, but without Laby-GI compressor whose task of gas compression onto the injection pressure is taken by the highpressure pump and an evaporator, a combination of MAN Diesel \& Turbo with the Cryostar EcoRel system for liquefaction and MDT with TGE system. MDT electronically controlled ME compared to traditional MC performance shows many advantages in optimization of the combustion process and a major step forward in improving combustion emissions. With the addition of gas, the results are even better.

MAN Diesel \& Turbo dual fuel two stroke diesel engines use for combustion natural gas, heavy or light diesel fuel. In heavy or light diesel fuel mode working principle is based on ME engine.

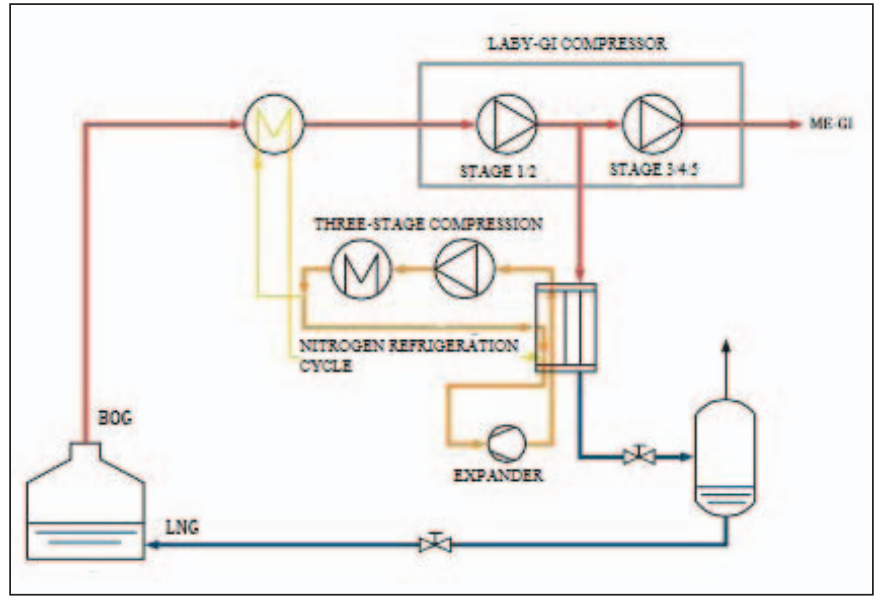

Figure 7 System LABY-GI MARK III [13]

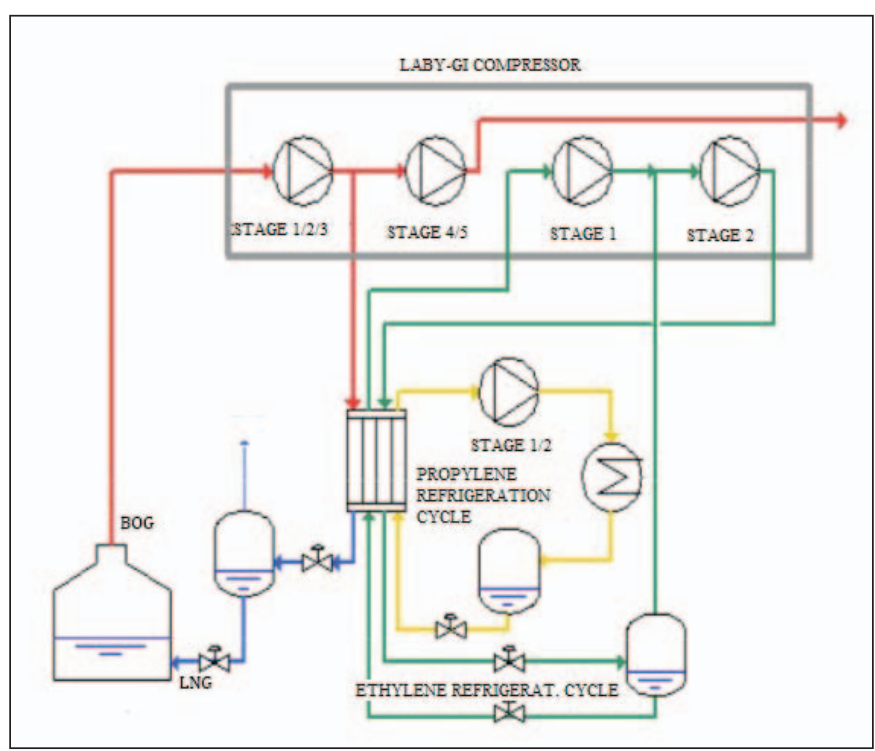

Figure 8 LABY-GI TGE [14]

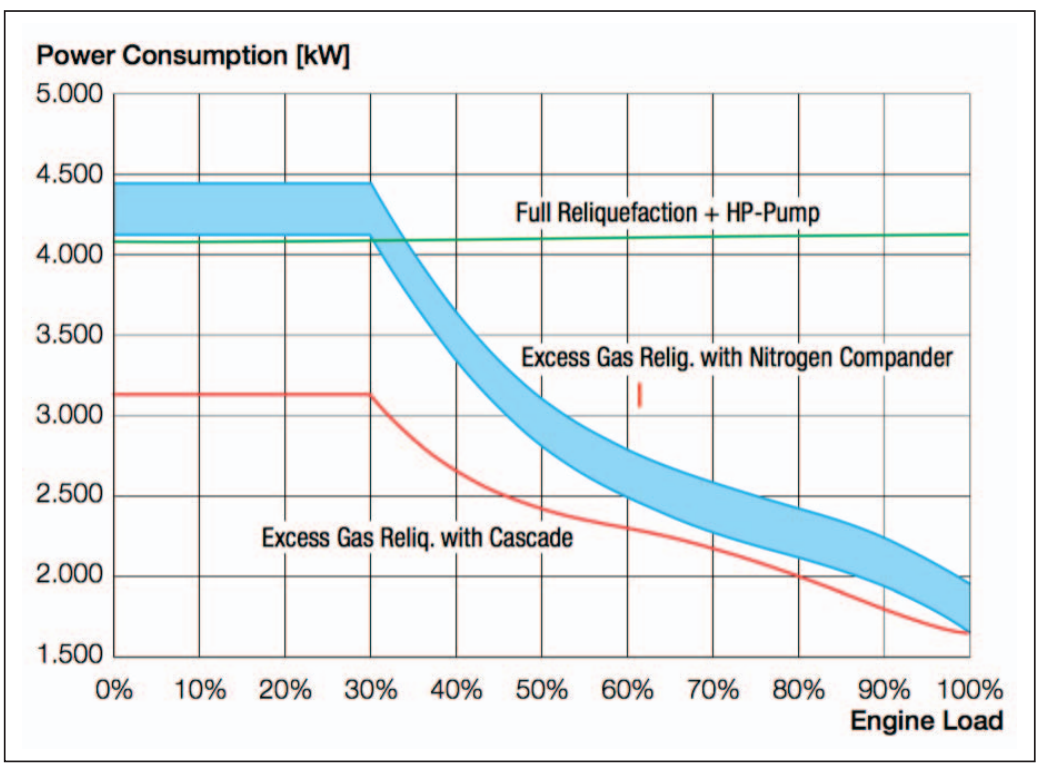

Figure 9 Comparison of power consumption when using different methods of liquefaction by changing the load of the main propulsion engine [12] 
The pilot oil mode is developed for gas operation and control system allows any ratio between fuel oil and gas, with a amount of fuel oil (pilot oil) of $3 \%$ at $100 \%$ engine load. Heavy fuel oil, marine diesel oil and marine gas oil can be used as pilot oil. Tests have shown that a pilot oil may be reduced to $1.5 \%$, however, the engine will then have some load limitation in the fuel oil mode [12].

The advantage of installing the Laby-GI compressor system and the high pressure LNG pump with a vaporizer, except for additional redundancy, provides a highly efficient gas supply system, especially if the dual fuel generators driven by gas and diesel fuel are installed.

The advantage of the multi-stage compressor Laby-GI is its compact design suitable for low inlet temperatures and different cylinder pressures. It is used to pressurize the boil-off natural gas, and pressurization of the cooling medium, as well as to achieve the natural gas pressure of 300 bar as needed for injection into a two-stroke engine system. This compressor incorporates all the compression functions required for the liquefaction system and those for the combustion engine.

Laby-GI MARK III system, shown schematically in Figure 7, is designed as a combination system for liquefaction of MARK III and the propulsion two-stroke engine that burns both gas and diesel. The system for liquefaction differs by boil-off gas cargo compressors that have been replaced by vertical reciprocating compressors Laby-GI. The compressor is a five-stage one, of which the first two stages are a part of the cargo liquefaction system (5-6 bar), and the other three raise the pressure by 300 bar for gas injection into the engine cylinder [13].

LABY-GITGE system, shown schematically in Figure 8, is intended for LNG ships of capacity between 170,000 and $210,000 \mathrm{~m}^{3}$, it is specific by the fact that it does not use nitrogen in its refrigeration cycle in the reverse Brayton cycle as other mentioned systems, but the combination of propylene and ethylene in the cascade cycle. This is because the thermodynamic properties are far better. The compressor is also a joint one for liquefying the boil-off gas and supplying the gas to the engine at a pressure of 150-300 bar [9].

For liquefaction, in three stages of compression the pressure from the cargo tank rises to 45 bar and condensed at $-100^{\circ} \mathrm{C}$ within a double compression of ethylene by an intermediate cooler. In the same heat exchanger ethylene is condensed at $-30^{\circ} \mathrm{C}$ and 19 bar by transferring the heat to the propylene cycle. The condensation of propylene at 17 bar and $40^{\circ} \mathrm{C}$ takes place in a closed cascade cycle with two degrees of compression by a separate screw compressor [9].

The use of a cascade system with two cooling mediums and the combustion of gas in a drive engine brings big savings compared with the use of the nitrogen refrigerant.

\section{Conclusion}

This overview shows the available technology of liquefaction, a combination with a propulsion dual-fuel electronically controlled slow rotating two-stroke diesel engine and trends in future development.

Brayton nitrogen refrigeration cycle is due to its ease of performance and robustness one of the most commonly used ones in ship application, despite the relatively high consumption of energy.

Area for further development and optimization lies in the application and development of single-cascade refrigeration systems and dual-fuel engines. This should be further contributed by a more refined regulation International Code of Safety for Ships Using Gases or Other Low Flashpoint Fuels- IGF Code expected in 2017 [15].

\section{References}

[1] Martinović, D.: Brodski rashladni uređaji, Školska knjiga, Zagreb, 1994.

[2] Sedlaczek R.: Boil-Off in Large and Small Scale LNG Chains, Diploma Thesis, Faculty of Engineering Science and Technology, Department of Petroleum Engineering and Applied Geophysics, Trondheim, 2008.

[3] Dr. K-D. Gerdsmeyer \& W.H. Isalski: On-board reliquefaction for LNG ships, Gastech 2005.

[4] Dobrota Đ., Lalić B., Komar I.: Problem of Boil-off in LNG Supply Chain.

[5] H.M. Chang, J.H. Park, K.S. Cha, S. Leeand K.H. Choe: Modified Reverse-Brayton Cycles for Efficient Liquefaction of Natural Gas.

[6] H.M. Chang, J.H. Par, S. Lee, and K.H. Choe: Combined BraytonJT Cycles with pure refrigerants for natural gas liquefaction.

[7] J.W. Moon, Y.P. Lee, Y.W. Jin, E.S. Hong, and H.M. Chang: Cryogenic Refrigeration Cycle for Re-Liquefaction of LNG Boil-Off Gas.

[8] Cryostar Magazine Br. 10, 2007.

[9] J. Romero Gómez, Ph.D., M. Romero Gómez, Ph.D., R. Ferreiro Garcia, Prof., A. De Miguel Catoira, Ph.D.: On board LNG reliquefaction technology: a comparative study, University of A Coruna, Spain.

[10] Hamworthy: LNG systems for marine application.

[11] Marek Matyszczak, Leszek Kaszycki: Characterization of the Liquefaction Systems installed on board of the LNG ships, Scientific Journals, Maritime University of Szczecin, 2011, 28(100), z. 1, pp. 83-87.

[12] MAN Diesel \& Turbo: ME-GI Dual Fuel, MAN B\&W Engines, A Technical, Operational and Cost-effective Solution for Ships Fuelled by Gas.

[13] Hamworthy: LNG Fuel Gas Systems.

[14] Burckhard Compression, TGE Marine: Efficient, Low Cost LNG BOG Handling by Integration of Cascade Liquifaction and Laby-GI Fuel Gas Compressor for ME-GI Propulsion System.

[15] ABS: LNG fuel ready vessels, December 2014. 